\title{
Utilização do Robot Operating System (ROS) em Conjunto com o Kit Didático LEGO Mindstorms no Ensino de Robótica Móvel
}

\author{
Carolina Fany Andreoni Duarte* Israel Filipe Silva Amaral* \\ Lorran Pires Venetillo Dutra* \\ Gilmar Pereira da Cruz Júnior ${ }^{* * *}$ Héctor Azpúrua ${ }^{* *, * * *}$ \\ Gustavo Medeiros Freitas ${ }^{*, * * *}$ \\ * Escola de Engenharia, Universidade Federal de Minas Gerais - \\ UFMG (e-mails: carol-duarte@ufmg.br, israelfilipe@ufmg.br, \\ lorranvenetillo@ufmg.br,gustavomfreitas@ufmg.br). \\ ** Dept. de Ciência da Computação, Universidade Federal de Minas \\ Gerais - UFMG. \\ *** Instituto Tecnológico Vale - ITV, (e-mail: hector.azpurua@itv.org). \\ **** Programa de Pós-Graduação em Engenharia Elétrica - \\ Universidade Federal de Minas Gerais - Av. Antônio Carlos 6627, \\ 31270-901, Belo Horizonte, MG, Brasil (e-mail: \\ gilmarpcjunior@ufmg.br)
}

\begin{abstract}
This paper presents a methodology for teaching mobile robotics for engineering courses at the Federal University of Minas Gerais (UFMG - Brazil). During the course "Mobile Robotics Laboratory", undergraduates use LEGO Mindstorms NXT robotics kits, sensors embedded in an Android cellular and the Robot Operating System (ROS) to implement and experimentally verify techniques regarding localization and mapping, sensory fusion and probabilistic filters. The activities are divided into three main practices based on a line follower robot, where students must: (i) calculate the path traveled using wheel odometry, (ii) fuse the results with cellular IMU data using Kalman filter (EKF), and (iii) perform the simultaneous localization and mapping (SLAM) combining data from a distance sensor through a RaoBlackwellized particle filter. The article presents theories addressed during the course, describing the practices proposed using ROS and the results obtained so far.

Resumo: Este artigo apresenta uma proposta de metodologia para ensino de robótica móvel para cursos de engenharia da Universidade Federal de Minas Gerais (UFMG). Durante a disciplina Laboratório de Robótica Móvel os alunos de graduação utilizam kits de robótica LEGO Mindstorms NXT, sensores embarcados em um celular com sistema operacional Android e o Robot Operating System (ROS) para aplicar na prática conhecimentos sobre modelos cinemáticos, fusão sensorial e filtros probabilísticos. As atividades são divididas em três principais práticas que têm como base um robô seguidor de linha, onde os alunos devem (i) calcular o caminho percorrido utilizando odometria das rodas, (ii) fundir os resultados com dados da IMU do celular utilizando filtro de Kalman (EKF), e (iii) realizar o mapeamento simultâneo (SLAM) do ambiente combinando os dados de um sensor de distância através de filtro de partículas Rao-Blackwellized. O artigo apresenta as teorias abordadas durante a disciplina, descrevendo as práticas propostas utilizando o ROS e os resultados obtidos até o momento.
\end{abstract}

Keywords: Mobile Robotics Education; Robot Operating System (ROS); LEGO Mindstorms NXT; Kalman Filter; Particle Filter.

Palavras-chaves: Educação em Robótica Móvel; Robot Operating System (ROS); LEGO

Mindstorms NXT; Filtro de Kalman; Filtro de Partículas. 


\section{INTRODUÇÃO}

Dispositivos robóticos vem sendo cada vez mais utilizados na indústria com o intuito de tornar os sistemas de produção mais robustos e flexíveis. Além disso, robôs têm sido aplicados com sucesso em diversas outras áreas nos últimos anos. No caso de robôs móveis, estes são também utilizados na exploração de regiões desconhecidas ou com riscos para a vida humana, por exemplo ambientes radioativos (Nagatani et al., 2013), exploração planetária (Ishigami et al., 2007) e mapeamento de minas abandonadas (Ferguson et al., 2004).

Considerando a importância da robótica nos dias atuais, a formação de profissionais capacitados a atuarem nessa área é de suma importância para o desenvolvimento econômico e social do país.

Uma das principais dificuldades no ensino de robótica consiste em associar os conceitos teóricos a experimentos tangíveis, em função da natureza multidisciplinar deste campo (Júnior et al., 2014). Compreender a complexidade de dispositivos robóticos e suas aplicações exige conhecimentos de elétrica e eletrônica, mecânica, sistemas, ciência da computação, economia e matemática (Spong et al., 2006). Porém, a abordagem predominantemente adotada em cursos de engenharia costuma fragmentar este conhecimento em disciplinas isoladas. Estas, se tratadas sem as interligações necessárias, podem levar o aluno a perder a noção do todo e não realizar a integração dos temas.

Uma forma de tornar efetiva a associação entre teoria e prática seria através de trabalhos de laboratórios motivadores (Wood, 2008). Entretanto, a realização de práticas com dispositivos robóticos reais nem sempre é algo fácil, dados os altos custos geralmente associados a estes dispositivos. Aliando isso à realidade financeira das universidades do Brasil, grande parte das instituições não consegue adquirir equipamentos a fim de enriquecer a experiência didática dos alunos. O ensino da robótica acaba se restringindo aos conceitos teóricos, tendo como resultado egressos com pouca ou nenhuma experiência prática.

Neste sentido, este artigo apresenta a proposta da disciplina Laboratório de Robótica Móvel, oferecida a alunos de graduação dos cursos de Engenharia de Controle e Automação, Elétrica e Sistemas da Universidade Federal de Minas Gerais (UFMG).

O objetivo da disciplina consiste em apresentar para os alunos de graduação conceitos teóricos relacionados à robótica móvel, incluindo introdução aos sensores e atuadores mais utilizados, arquiteturas comuns de locomoção e seus respectivos modelos cinemáticos, estratégias de planejamento de caminhos e controle de navegação, além de técnicas probabilísticas para localização e mapeamento.

Por se tratar de uma disciplina laboratorial, o foco principal é possibilitar aos alunos implementar e analisar as teorias apresentadas através de experimentos com robôs reais. Buscando diminuir os custos associados e permitir

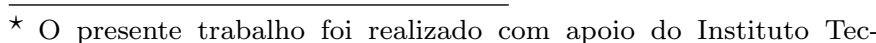
nológico Vale (ITV) e do Conselho Nacional de Desenvolvimento Científico e Tecnológico ( $\mathrm{CNPq})$. que a metodologia de ensino proposta possa ser empregada em outras instituições de ensino, as práticas sugeridas utilizam equipamentos de baixo custo e fácil acesso, no caso kits didáticos de robótica LEGO Mindstorms NXT e celulares com sistema operacional Android. Os trabalhos propostos são implementados utilizando pacotes do Robot Operating System (ROS), um software livre amplamente utilizado pela comunidade de robótica (Quigley et al., 2009). De fato, a apresentação e familiarização dos alunos com o ROS também constitui um objetivo secundário da disciplina.

As atividades experimentais propostas aos alunos são divididas em três principais práticas que têm como base um robô seguidor de linha, onde os alunos devem (i) calcular o caminho percorrido utilizando odometria das rodas, (ii) fundir os resultados com dados da IMU do celular utilizando filtro de Kalman (EKF), e por fim (iii) realizar a localização e mapeamento simultâneos (SLAM) do ambiente combinando os dados de um sensor de distância através de filtro de partículas Rao-Blackwellized.

Cabe ressaltar que todas as práticas sugeridas, tutoriais e códigos utilizados para a implementação da disciplina estão disponíveis online, buscando facilitar a adoção da metodologia didática proposta em outras universidades e instituições de ensino.

Este artigo descreve na Seção 2 o hardware e software utilizados nas práticas propostas, junto a metodologia didática empregada. A Seção 3 apresenta a parte teórica abordada durante a disciplina, assim como sua implementação utilizando pacotes do ROS. Na Seção 4 são destacados os resultados obtidos durantes as práticas, junto com análises sugeridas aos alunos. Por fim, a Seção 5 apresenta conclusões e sugestões de trabalhos futuros.

\section{MATERIAIS E MÉTODOS}

A seguir são apresentados maiores detalhes sobre o hardware e o software utilizados pelos alunos, além da metodologia de ensino empregada em sala de aula.

\subsection{Hardware}

Durante as aulas de Laboratório de Robótica Móvel, foi utilizado o kit de robótica programável LEGO Mindstorms NXT. Além das várias peças para a construção mecânica de um robô, o kit conta com um processador ARM7TDMI de 32-bit e um microcontrolador Atmel AT91SAM7S256 com $256 \mathrm{~KB}$ de memória flash e $64 \mathrm{~KB}$ de memória RAM, além de suporte para bluetooth. A alimentação pode ser feita através de pilhas ou de uma bateria de LiIon recarregável (utilizada neste projeto). O valor da nova versão do kit didático LEGO Mindstorms é de aproximadamente USD 350,00.

É possível programar o processador em diversas linguagens, como por exemplo $\mathrm{C} \#, \mathrm{C}++$ e Ruby. Outra forma comum de programar o NXT é através de módulos de integração entre Matlab e ROS. A versão NXT do kit LEGO não possibilita embarcar o ROS no processador; 
assim, é preciso que o robô esteja sempre conectado com o computador via cabo USB.

O LEGO NXT é capaz de receber dados de até quatro sensores e comandar três motores através de cabos RJ12. Cinco dos sensores mais utilizados são:

- Sensor de toque - Retorna um valor binário, 0 se não estiver sendo pressionado, e 1 caso o contrário;

- Sensor de luz - Este sensor pode detectar a luz refletida de um LED autocontido, ou então a luz ambiente;

- Sensor de som - Usado para medir a intensidade sonora do ambiente ao seu redor em dB ou em dBA (frequências por volta de $3-6 \mathrm{kHz}$, as quais o ouvido humano é mais sensível);

- Sensor ultrassônico - Usado para medir distâncias de objetos posicionadas entre 0 e $255 \mathrm{~cm}$ do sensor, com uma precisão de +/- 3cm;

- Sensor de rotação - Corresponde a um encoder rotacional óptico, que mede a rotação do motor com exatidão de 1 grau.

Para auxiliar os estudantes da disciplina na montagem do robô, foi utilizado o programa LEGO Digital Designer (https : //www.LEGO.com/en - us/ldd/download). Este é um software gratuito desenvolvido pela LEGO, que permite a montagem e visualização de construções virtuais usando peças padronizadas. O programa possibilita também a criação de tutorias de construção passo-a-passo, facilitando o processo de montagem do robô. A Figura 1 mostra o modelo sensor_car, que é umas das montagens utilizadas na disciplina.
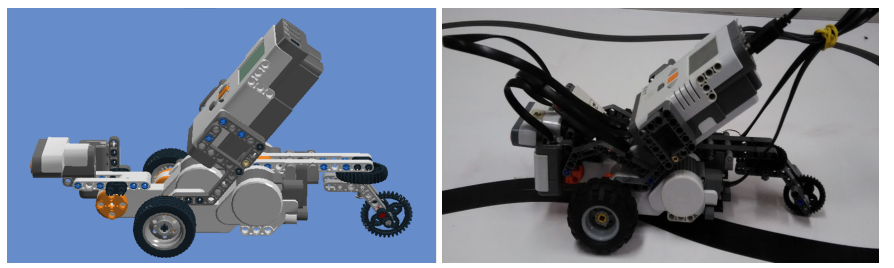

Figura 1. sensor_car no programa LEGO Digital Designer à esquerda, e montagem realizada à direita.

A fim de expandir as possibilidades com o kit LEGO, é utilizado um celular com sistema operacional Android, que conta com diversos sensores embarcados, tais como acelerômetro, giroscópio, GPS e barômetro. O modelo do celular deve ser observado, pois ele pode possuir diferentes sensores embarcados. Na disciplina, o acelerômetro e o giroscópio do celular são utilizados para reproduzir uma IMU (em inglês Inertial Measurement Unit) fornecendo dados sobre a orientação do dispositivo.

O mapeamento do ambiente é realizado através de um instrumento para medir distâncias. A primeira alternativa seria utilizar o sensor ultrassônico do kit LEGO. Entretanto, o dispositivo apresenta baixa acurácia, acarretando em erros nos mapas gerados. A forma mais rápida de solucionar o problema foi empregar um laser range finder Hokuyo URG-04LX-UG0 disponível na UFMG. Este sensor, que utiliza o tempo de voo de um feixe de luz como princípio de funcionamento, possui campo de visão de $240^{\circ}$ e distância de detecção de até $5.6 \mathrm{~m}$. Cabe ressaltar que apesar dos altos custos normalmente associados aos sensores laser, já se encontram no mercado alguns dispositivos de baixo custo (USD 100,00), por exemplo o RPLidar A1M8 ou o YDLIDAR X4 $360^{\circ}$.

\subsection{Software}

O ROS é um meta Sistema Operacional (SO) open-source executado sobre outro SO, geralmente Ubuntu, para atuar como plataforma de desenvolvimento de algoritmos para aplicação em robótica. O sistema consiste numa coleção de ferramentas, bibliotecas e convenções que tem como objetivo simplificar a criação de sistemas robóticos, promovendo a reutilização de código e a cooperação em uma comunidade de programadores. Para tal, o ROS possui características como abstração de camada de hardware, implementações simplificadas para funções de uso comum, pacotes e gestão da comunicação entre processos.

O ROS permite que diferentes pacotes sejam integrados de forma simples, dando um nível de abstração aos processos mais próximos do hardware e facilitando a implementação de projetos em grande escala. Os pacotes são a unidade principal para organizar o software no ROS. Cada pacote pode conter processos (nós) de tempo de execução, bibliotecas dependentes do ROS, conjuntos de dados e arquivos de configuração. O ROS é projetado para funcionar de forma modular, onde os nós são processos que executam tarefas em paralelo. O alto nível de modularização entre as diferentes funcionalidades possibilita que mesmo no caso de uma falha - um nó pare de funcionar, o sistema como um todo não seja comprometido.

A comunicação entre nós e feita através da troca de mensagens, que correspondem a estruturas padronizadas de dados. Cada mensagem é transmitida por meio de um sistema de comunicação com semântica de publicação e inscrição.

Durante as aulas da disciplina de Laboratório de Robótica Móvel, foi utilizada a distribuição Kinetic Kame, executada no Ubuntu 16.04 (Xenial). A recomendação para os alunos é instalar o ROS numa partição do computador e utilizar um gerenciador de boot, por exemplo o GRUB, ao invés de rodar o ROS numa máquina virtual.

No caso do kit didático, a programação do controlador NXT pode ser feita através de diversos programas, incluindo o ROS. Um pacote para fazer a comunicação do NXT com o ROS já é disponibilizado online; entretanto, este pacote foi desenvolvido para uma versão antiga do software (ROS Electric - atualmente sem suporte). Como a versão utilizada durante as aulas é o ROS Kinetic, foi necessário fazer uma adaptação no código, tornando possível a utilização desse pacote nas versões mais recentes do ROS. Foram realizadas melhorias significativas no pacote: atualização das bibliotecas usadas para conectividade serial e bluetooth, adição da captura e interpretação de dados do sensor de áudio, modificação do plugin de visualização dos sensores ultrassônicos para funcionar em versões recentes do simulador Gazebo, e várias melhorias na parte do controle diferencial e a forma de compilação do pacote. A nova versão do pacote está disponível no repositório ros_LEGO_nxt (https : //github.com/h3ct0r/ros_LEGO_nxt). 
Para que os sensores embarcados num celular Android sejam lidos no ROS, o aplicativo ROS_All_Sensors_Driver (disponível em https : //play.google.com/store/apps/det ails?id = org.ros.android.android_all_sensors_driver) deve ser instalado em um celular conectado com o ROS via rede WiFi. Uma vez conectado, é possível posicionar o celular no robô e obter sua posição, via GPS (em ambientes abertos), e orientação através dos dados dos giroscópios e acelerômetros embarcados.

\subsection{Metodologia Didática Proposta}

A disciplina de Laboratório de Robótica Móvel é organizada em módulos teóricos, onde são apresentados aos alunos os conceitos básicos de robótica móvel, intercalados de módulos práticos onde estes têm a oportunidade de implementar e analisar as teorias abordadas.

A primeira atividade proposta aos alunos consiste em implementar com o LEGO e o ROS um dispositivo robótico seguidor de linha (Pakdaman and Sanaatiyan, 2009; Kaiser et al., 2014; Hasan et al., 2012). Esta é uma aplicação bastante comum em robótica móvel, cujo objetivo é utilizar os sensores de luz para detectar uma linha preta sobre uma superfície clara, e comandar os motores do robô de forma a seguir o caminho, neste caso formando um circuito fechado, conforme ilustrado na Figura 2. A implementação do seguidor de linha serve como base para as práticas subsequentes, e possibilita aos alunos um primeiro contato com o hardware e software utilizados na disciplina.

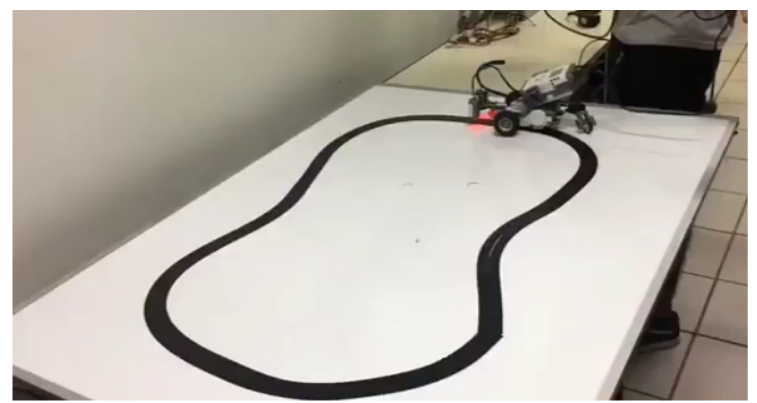

Figura 2. Circuito formado por linha preta a ser seguida pelo robô.

Após a implementação do seguidor de linha, os alunos devem realizar mais três atividades práticas adicionando funcionalidades ao dispositivo. A primeira prática consiste em calcular o caminho percorrido pelo robô seguidor de linha utilizando apenas os dados de leitura dos encoders. A segunda prática consiste em melhorar os resultados obtidos utilizando também os sensores embarcados no celular de forma semelhante a uma IMU, fundindo os dados através de um Filtro de Kalman Estendido (EKF). A última prática consiste em, adicionando um sensor de distância ao conjunto, calcular a localização e mapear os arredores da pista simultaneamente enquanto o robô segue a linha preta; neste caso, a fusão dos dados é realizada utilizando um filtro de partículas Rao-Blackwellized.

As atividades práticas são realizadas pelos alunos organizados em grupos de duas ou três pessoas. Durante as práticas, são fornecidos diferentes materiais de referência, incluindo tutoriais em textos e vídeos, disponíveis em https : //github.com/israelfi/Tutoriais - Laboratorio-
Robotica - Movel, contendo instruções detalhadas sobre a instalação e utilização dos pacotes de ROS necessários.

Ao final da disciplina cada grupo de alunos entrega um relatório em formato de artigo científico, escrito em $\mathrm{IAT}_{\mathrm{E}} \mathrm{X}$, descrevendo os conceitos teóricos estudados, as práticas realizadas e os resultados obtidos. Além disso, os alunos devem fazer uma apresentação oral do trabalho realizado, também no formato de congresso científico, com 15 minutos para apresentação e 5 minutos para perguntas.

\section{PRÁTICAS PROPOSTAS}

\subsection{Localização com Odometria}

A odometria consiste no desenvolvimento de um modelo matemático que descreve como os movimentos de certas juntas produzem movimento no robô e, então, integrar esses dados ao longo do tempo a fim de se obter a posição e orientação do robô (Dudek and Jenkin, 2010).

Um robô móvel com arquitetura diferencial pode ser modelado com relativa facilidade a partir do modelo básico de um monociclo, que assume as seguintes hipóteses (Siciliano and Khatib, 2016):

- Locomoção numa superfície plana;

- Roda posicionada de forma perpendicular ao solo;

- Rotação pura entre roda e terreno, sem deslizamento;

- Roda corresponde a um corpo rígido sem deformação.

A pose $x$ de um robô móvel planar é definida por:

$$
x=\left[x_{r}, y_{r}, \theta_{r}\right]^{T}
$$

onde $x_{r}$ e $y_{r}$ representam a posição planar e $\theta_{r}$ a orientação com respeito ao sistema de coordenadas inicial. O modelo da cinemática diferencial $\dot{x}=J(\theta) \dot{\theta}$ do monociclo é dado por:

$$
\left[\begin{array}{c}
\dot{x}_{r} \\
\dot{y}_{r} \\
\dot{\theta}_{r}
\end{array}\right]=\left[\begin{array}{cc}
\cos (\theta) & 0 \\
\sin (\theta) & 0 \\
0 & 1
\end{array}\right]\left[\begin{array}{c}
v \\
\omega
\end{array}\right]
$$

onde $\dot{x}$ e $\dot{y}$ são as velocidades lineares na direção $x$ e $y$ do referencial inercial, $v$ é a velocidade linear, $\omega$ é a velocidade angular, e $J$ é a matriz Jacobiana.

Durante a disciplina de Laboratório de Robótica Móvel, são utilizados robôs com uma arquitetura diferencial (Figura 3), onde:

- Não existem rodas diretrizes;

- A mudança de direção ocorre modificando as velocidades relativas entre as rodas da direita e da esquerda;

- O movimento angular ocorre (teoricamente) sem escorregamento.

Considerando a arquitetura diferencial, $v$ e $\omega$ podem ser encontrados pelas Equações 3 e 4, respectivamente:

$$
\begin{aligned}
& v(t)=\frac{v_{D}(t)+v_{E}(t)}{2} \\
& \omega(t)=\frac{v_{D}(t)-v_{E}(t)}{L}
\end{aligned}
$$



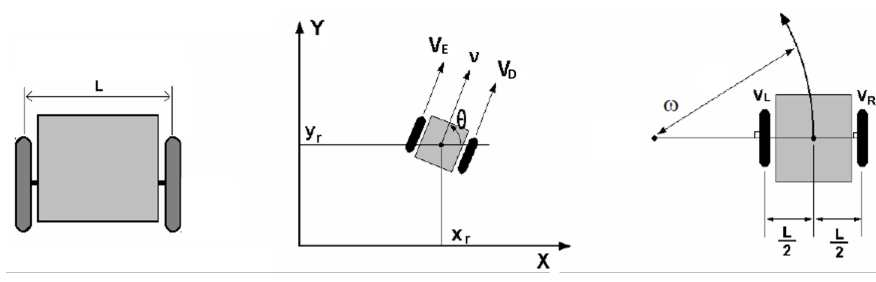

Figura 3. Robô móvel com arquitetura diferencial.

onde $L$ é a distância entre as rodas e $v_{D}$ e $v_{E}$ são as velocidades lineares das rodas direita e esquerda, calculadas a partir da leitura dos encoders.

É possível utilizar integração numérica de primeira ordem, através do método de Euler, para calcular a pose a partir da cinemática diferencial:

$$
\left[\begin{array}{c}
x_{r}(k+1) \\
y_{r}(k+1) \\
\theta_{r}(k+1)
\end{array}\right]=\left[\begin{array}{c}
v(k) \cos (\theta) \Delta t+x_{r}(k) \\
v(k) \sin (\theta) \Delta t+y_{r}(k) \\
\omega(k) \Delta t+\theta_{r}(k)
\end{array}\right]
$$

no qual $x_{r}(k), y_{r}(k), \theta_{r}(k)$ correspondem a estimação da pose no instante de tempo $k$, e $x_{r}(k+1), y_{r}(k+1), \theta_{r}(k+1)$ são a estimação da pose no instante de tempo $k+1$.

Observando (5) é possível perceber que a exatidão da estimação da pose é proporcional ao inverso da taxa de amostragem $\Delta t$. Por causa disso, esta abordagem pode acarretar muitos erros no cálculo da pose, que vão se acumulando ao longo do tempo. Esse fenômeno é retratado na Figura 4.

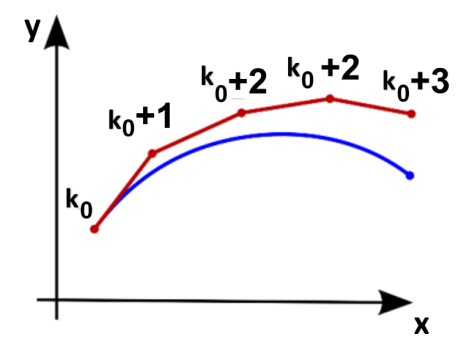

Figura 4. Comparação entre o percurso real do robô em azul e o percurso dado pela integração numérica de primeira ordem em vermelho.

A implementação da Localização com Odometria foi feita

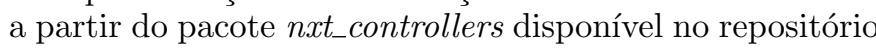
ros_LEGO_nxt.

\subsection{Localização com Filtro de Kalman}

O método de localização com odometria consiste em integrar as medidas de velocidades feitas com os encoders dos motores e, assim, estimar a pose do robô. Porém, as medidas de odometria são ruidosas e comprometidas por escorregamento das rodas, de forma que a navegação estimada inevitavelmente diverge do percurso verdadeiro (Thrun and Leonard, 2008).

Uma maneira de minimizar o erro da estimativa consiste em utilizar outros sensores e uma estratégia probabilística de fusão de dados, por exemplo, o Filtro de Kalman Estendido (do inglês - Extended Kalman Filter - EKF). O EKF provê, de forma eficiente, um método recursivo de estimar o estado de um sistema dinâmico na presença de ruídos (Choset et al., 2005). O fato dele ser recursivo significa que é preciso somente a estimativa anterior e a atuação ou medição atual para se realizar a estimativa do estado atual.

Geralmente, métodos de estimação probabilística podem ser descritos como processos de duas etapas: predição e correção. Na etapa de predição é levado em conta a estimativa do estado anterior mais a ação de controle para estimar o estado atual; por não usar nenhuma observação do estado no tempo atual, ela é chamada de estimativa a priori. Já na etapa de correção, a estimativa a priori é combinada com a observação do estado atual; o resultado da etapa de correção é referida também como estimativa a posteriori.

O Filtro de Kalman Estendido pode ser usado em sistemas não lineares representados como:

$$
\begin{gathered}
x(k+1)=f(x(k), u(k), k)+v(k) \\
y(k)=h(x(k), k)+w(k)
\end{gathered}
$$

no qual $x \in \mathbb{R}^{n}$ denota o valor da estimativa do estado, $y \in \mathbb{R}^{p}$ é a saída contendo os dados dos sensores, $u \in \mathbb{R}^{m}$ é o vetor usado para representar as entradas do sistema, por exemplo os comandos de velocidades. $v \in \mathbb{R}^{n}$ é o ruído do processo com matriz de covariância $V(k)$, e $w \in \mathbb{R}^{p}$ é a medida de ruído com matriz de covariância $W(k)$. No caso do robô diferencial, $f(x(k), y(k), k)$ equivale à $(5)$.

É assumido que:

$$
f: \mathbb{R}^{n} \times \mathbb{R}^{m} \times \mathbb{Z}^{+} \rightarrow \mathbb{R}^{n}
$$

e

$$
h: \mathbb{R}^{n} \times \mathbb{Z}^{+} \rightarrow \mathbb{R}^{p}
$$

são diferenciáveis em $x(k)$. Uma maneira de realizar a estimação de estado para sistemas deste tipo através do Filtro de Kalman Estendido é linearizar as equações da estimativa atual.

As equações do Filtro de Kalman Estendido podem ser resumidas da seguinte maneira:

- Etapa de predição: O estado do sistema é representado por $x(k)=\left[x_{r}(k), y_{r}(k), \theta_{r}(k)\right]^{T}$ no instante $k$. A entrada do sistema $u(k)=\left[u_{1}(k) ; u_{2}(k)\right]^{T}$ corresponde às velocidades linear $v(k)$ e angular $\omega(k)$. A pose do veículo é estimada pelo sistema não linear:

$$
\begin{gathered}
\hat{x}(k+1 \mid k)=f(\hat{x}(k \mid k), u(k), k) \\
P(k+1 \mid k)=F(k) P(k \mid k) F(k)^{T}+V(k)
\end{gathered}
$$

no qual $\hat{x}(k+1 \mid k)$ é a estimativa do estado para o instante $k+1$ dada em relação ao instante $k ; P(k+1 \mid k)$ é a matriz de covariância em $k+1$ dada em relação a $k ; P(k \mid k)$ é a matriz de covariância do erro a priori; e $V(k)$ é a matriz de covariância do ruído de processo; $F(k)$ é a matriz que codifica a dinâmica do sistema, determinada a partir da linearização da função $f(x(k), u(k), k)$ em relação aos estados $x(k)$, conforme (10):

$$
F(k)=\left.\frac{\partial f}{\partial x}\right|_{x=\hat{x}(k \mid k)}
$$

- Etapa de correção: Após estimar $\hat{x}(k+1 \mid k)$, é possível corrigir a pose do veículo baseado na saída $y(k+1 \mid k)$ para 
gerar a próxima estimativa de estado $\hat{x}(k+1 \mid k+1)$. As equações da etapa de correção são:

$$
\begin{gathered}
\hat{x}(k+1 \mid k+1)=\hat{x}(k+1 \mid k)+R v \\
P(k+1 \mid k+1)=P(k+1 \mid k)-R H(k+1) P(k+1 \mid k)
\end{gathered}
$$

Sendo que

$$
\begin{gathered}
v=y(k+1)-h(x(k+1 \mid k), k+1) \\
R=P(k+1 \mid k) H(k+1)^{T} S^{-1} \\
S=H(k+1) P(k+1 \mid t) H(k+1)^{T}+W(k+1)
\end{gathered}
$$

no qual $\hat{x}(k+1 \mid k+1)$ é a estimativa dos estados no instante $k+1 ; R$ é a fator de ponderação de $v$ entre a estimativa prevista $h(x(k+1 \mid k), k+1)$ e as medidas ruidosas $y(k+1)$; $P(k+1 \mid k+1)$ é a matriz de covariância para o instante $k+1 ; W(k+1)$ é a matriz de covariância referente aos ruídos dos sensores; $H(k+1)$ é a matriz que descreve como os estados são mapeados na saída, determinada a partir da linearização da função $h(x(k+1 \mid t), k+1)$ em relação aos estados $x(k)$, conforme (16):

$$
H(k+1)=\left.\frac{\partial h}{\partial x}\right|_{x=\hat{x}(k+1 \mid k)}
$$

A prática proposta aos alunos da disciplina de Laboratório de Robótica Móvel consiste em realizar a localização do robô enquanto ele estiver seguindo a linha de um percurso fechado conforme ilustrado na Figura 2, fazendo uso do Filtro de Kalman. Para implementar o filtro com o ROS, foi utilizado o pacote robot_pose_ekf (disponível no link https : //github.com/ros - planning/robot_pose_ekf). Este pacote é usado para estimar a pose 3D do robô baseado nas medições de odometria por encoder, IMU (no caso da disciplina, foram utilizados os dados de giroscópios e acelerômetros embarcados um celular Android) e odometria visual (este último não foi utilizado na disciplina). Os tópicos subscritos pelo pacote são:

- /odom: Este tópico contém informações sobre a pose 2D do robô vindas dos encoders. A pose $2 \mathrm{D}$ contém a posição e orientação do robô no plano do chão e a covariância desta pose. A partir dos dados deste tópico é feita a etapa de predição;

-/imu_data: Neste tópico, há informações sobre a orientação 3D (representada através dos ângulos de roll, pitch e yaw)fornecidas por uma IMU. Como a IMU tem uma referência de gravidade, os ângulos roll e pitch são considerados como absolutos e o ângulo yaw é interpretado como ângulo relativo. Para especificar a incerteza na medição da orientação é usada uma matriz de covariância. A partir dos dados deste tópico são feitos os cálculos da etapa de correção.

Vale ressaltar que para o pacote robot_pose_ekf funcionar, não é preciso que os três tópicos estejam disponíveis sempre. A saída do filtro contendo a pose 3D estimada do robô é publicada no tópico:

\section{-/robot_pose_ekf/odom_combined}

\subsection{Localização e Mapeamento Simultâneos com Filtro de Partículas}

A construção de mapas é uma das tarefas fundamentais para robôs móveis, e em muitos casos está associada à localização (Grisetti et al., 2007). O problema de localização e mapeamento simultâneos, conhecido como SLAM (Simultaneous Localization and Mapping), consiste no fato de que para localizar o robô em um ambiente necessita-se de um mapa, e para construir um mapa é necessária a localização precisa do robô. As principais abordagens para a solução são métodos utilizando o EKF, no qual são calculados de forma conjunta as estimativas sobre a localização e o mapa, métodos de Otimização Global baseados em energia, e métodos utilizando filtros bayesianos recursivos como filtros de partículas, que utilizam amostragens para definir uma grandeza.

O custo computacional do filtro de partículas é alto devido ao seu funcionamento recursivo. Uma das técnicas aplicadas para reduzir este custo é o filtro de partículas RaoBlackwellized proposto em (Murphy, 2000; Doucet et al., 2000). A abordagem utiliza um conjunto de partículas para determinar a estimativa a posteriori sobre os caminhos do robô. Para cada caminho é associado um mapa individual calculado de forma analítica, supondo que esse seja verdadeiro.

O algoritmo do filtro de partículas Rao-Blackwellized inicia a partir da pose do robô na origem do mapa $x(0)=$ $\left[\begin{array}{lll}0 & 0 & 0\end{array}\right]^{T}$. Um conjunto $M$ de partículas $j$, que representam os caminhos do robô $x_{j}$, é determinado a partir da pose inicial.

Inicialmente é associado um mapa a cada partícula, simplificando o problema à estimação da localização. Dados os sinais de controle $u(1: k)$, as medições dos sensores $y(1: k)$ e o mapa $m_{j}$, utiliza-se o filtro de partículas para calcular a estimativa a posteriori para os caminhos, conforme (17):

$$
\begin{aligned}
& P(x(1: k), m \mid u(0: k-1), y(1: k))= \\
& P(m \mid x(1: k), y(1: k)) P(x(1: k) \mid y(1: k), u(0: k-1))
\end{aligned}
$$

no qual o termo do lado esquerdo é a estimativa a posteriori, $P(m \mid x(1: k), y(1: k))$ é a estimativa do mapa condicionado aos sinais de controle em conjunto com as medidas dos sensores, e $P(x(1: k) \mid y(1: k), u(0: k-1))$ é a estimativa da medidas dos sensores condicionada aos sinais de controle.

Através do modelo de movimentação do robô e da probabilidade a posteriori são calculadas as próximas localizações para cada partícula $x_{j}^{\prime}$. Supondo que as novas posições são as verdadeiras, agora o problema se resume ao mapeamento, determinando o incremento do mapa $m_{j}$ dadas as probabilidades das observações $y(k)$, de acordo com (18), normalizadas para cada partícula $j$ :

$$
\left.m_{j}(1: k-1)=\underset{m}{\operatorname{argmax}} P\left(m \mid x_{(j)}\right)(1: k), y(1: k-1)\right)
$$

O resumo do algoritmo do SLAM utilizando o filtro de partículas Rao-Blackwellized pode ser melhor compreendido através do Algoritmo 1, no qual os parâmetros de entrada são os sinais de controle $u(0: k-1)$, as medidas de observação $y(1: k)$ e o conjunto $M$ de $N$ partículas definidas por uma probabilidades $x_{j}$ e um fator de peso $w_{j}$. O parâmetro de saída é o conjunto $M^{\prime}$ com as estimativas a posteriori $P(x(1: k), m \mid u(0: k-1), y(1: k))$ para cada partícula re-amostrada. $O$ conjunto $M$ é re-amostrado de forma a eliminar as amostras de menor fator de peso $w_{j}$. O 
procedimento ocorre de forma iterativa a cada incremento dado por uma nova movimentação do robô ou realizando novas leituras dos sensores.

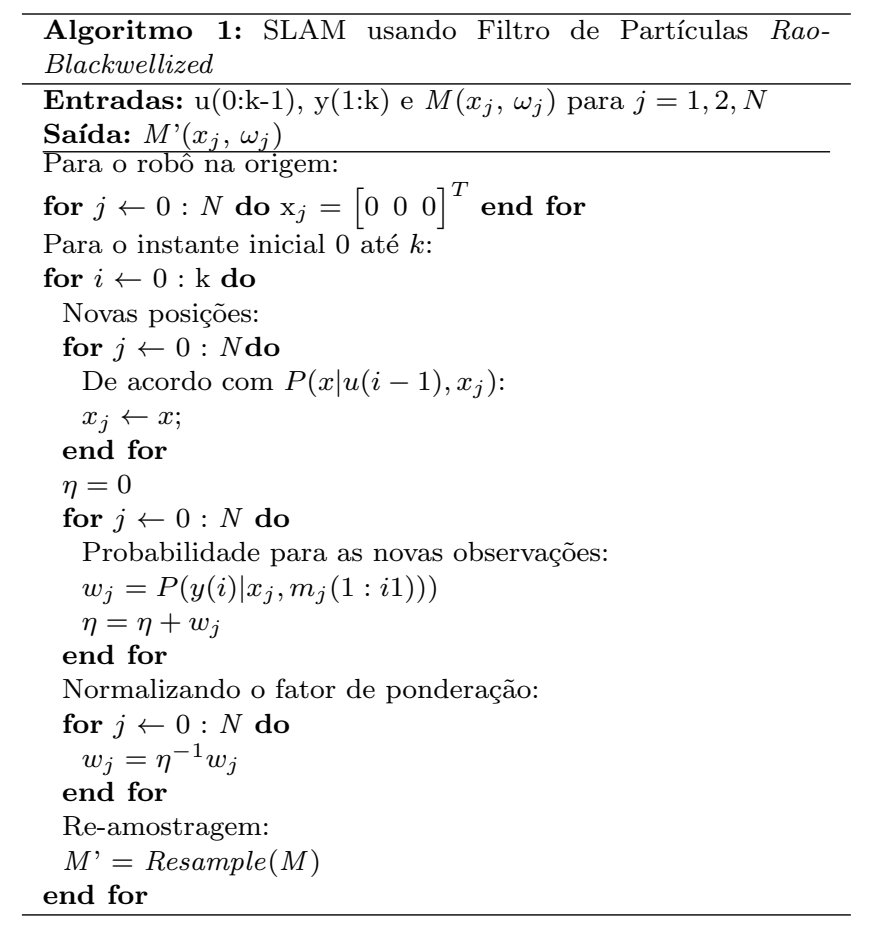

Durante a disciplina de Laboratório de Robótica Móvel os alunos devem realizar a localização do robô enquanto ele estiver seguindo um percurso fechado e, ao mesmo tempo, mapear o ambiente ao redor com o auxílio de um sensor de distância, no caso um laser Hokuyo. Para tal, foi utilizado o pacote gmapping (disponível no link https : //github.com/OpenSLAM - org/openslam_gmapping). Este pacote implementa um filtro de partículas RaoBlackwellized para mapear a região visível pelo laser e estimar a pose do robô nessa região, baseado nas medidas do laser e odometria.

Para a realização desta prática, foram adicionadas na pista paredes de papelão com a finalidade de delimitar o mapa. Também foram introduzidos no centro da pista dois objetos com formatos diferentes, buscando auxiliar na localização do robô e tornar o mapa mais representativo.

Os tópicos subscritos pelo pacote são:

- Itf: Este tópico contém a transformada necessária para relacionar os frames do laser e da odometria. É a partir dessa transformada que o filtro acessa os dados da odometria. Caso estes não estejam sendo publicados, o filtro não funcionará de forma correta e o mapa estará comprometido;

- /scan: Neste tópico estão as medidas do laser que serão usadas para criar o mapa. Cada medida é um vetor contendo as medições da distância para cada ângulo do laser.

Os dados do mapa são publicados nos seguintes tópicos:

- /map: Este tópico contém um vetor com uma posição para cada ponto do laser, e com ele é gerado o mapa. Contém também os principais parâmetros utilizados, tais como resolução, origem e o tamanho do mapa;
- /map_metadata: Este tópico contém os parâmetros do mapa, tornando possível acessá-los de forma mais rápida.

\section{RESULTADOS}

Esta seção apresenta os resultados que os alunos devem obter após a realização das práticas propostas. Além da experiência adquirida durante a implementação dos filtros de Kalman e partículas utilizando pacotes do ROS, os alunos podem também analisar os dados obtidos, fazendo uma comparação com a teoria ministrada em sala de aula.

O cálculo da pose através de dados de odometria por encoders é impreciso, principalmente durante movimentos de rotação do robô. Simplificações não atendidas (e.x. escorregamento das rodas) e problemas de integração numérica vão gerando erros acumulados; este comportamento fica mais evidenciado durante o seguimento de um caminho fechado, conforme ilustrado na Figura 5(a). Os erros acumulados são suavizados de forma considerável com a fusão por EKF dos dados de orientação fornecidos por sensores embarcados num celular Android. A mesma Figura apresenta a estimação da pose através do filtro de Kalman, enquanto o robô percorre o mesmo circuito fechado: apesar dos erros ainda presentes, o resultado é visivelmente mais consistente que o obtido com a utilização apenas dos encoders.

Já a utilização do filtro de partículas Rao-Blackwellized fornece um mapa da pista percorrida, criado com base nas medidas do laser e na odometria do robô. Nas Figuras 5(b) e (c) é possível visualizar a pista real e o mapa gerado. Na Figura 5(c), os pontos demarcados como obstáculos são representados por quadrados pretos, enquanto a área cinza claro é o espaço visualizado sem obstáculos. As bordas da imagem, em cinza escuro, são zonas desconhecidas pelo robô. Na imagem também são visíveis diversos pontos brancos; eles correspondem a medições do laser naquele instante de tempo.

\section{CONCLUSÃO}

Este artigo apresentou uma proposta de metodologia de ensino sobre robótica móvel para cursos de graduação na área de engenharia, implementando e verificando conceitos teóricos sobre modelos cinemáticos, fusão sensorial e filtros probabilísticos através da utilização de kits de robótica LEGO Mindstorms NXT, sensores embarcados num celular Android e de distância, e o Robot Operating System (ROS).

Partindo da implementação de um robô seguidor de linha, os alunos da disciplina realizam três atividades práticas com objetivos de (i) calcular o caminho percorrido utilizando odometria das rodas, (ii) fundir os resultados com dados da IMU do celular utilizando filtro de Kalman (EKF), e (iii) realizar o mapeamento simultâneo (SLAM) do ambiente combinando os dados de um sensor de distância através de filtro de partículas Rao-Blackwellized.

O hardware utilizado durante as práticas pode ser considerado de baixo custo, em comparação com outras plataformas didáticas de robótica, de forma a facilitar a adoção da metodologia proposta em outras universidades e instituições de ensino. Neste sentido, todas as práticas sugeridas e 


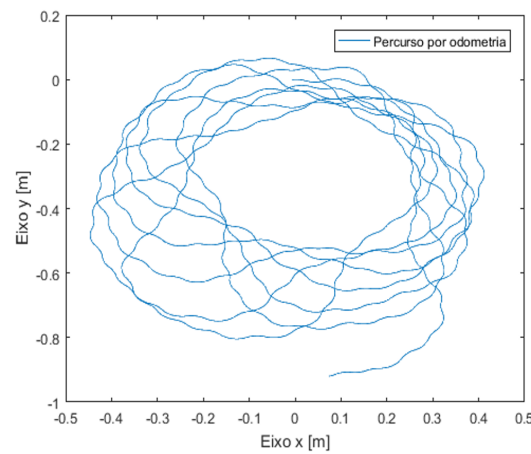

(a) Localização obtida com odometria por encoder e com fusão de dados via EKF.

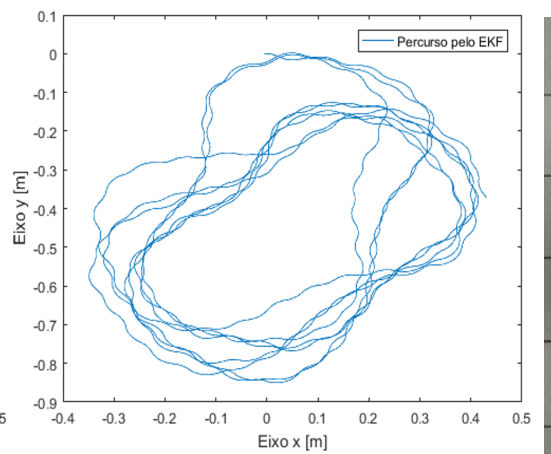

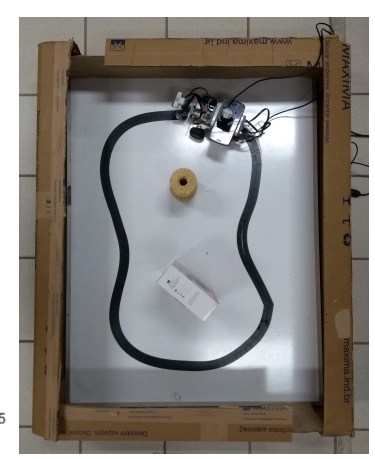

(b) Pista real.

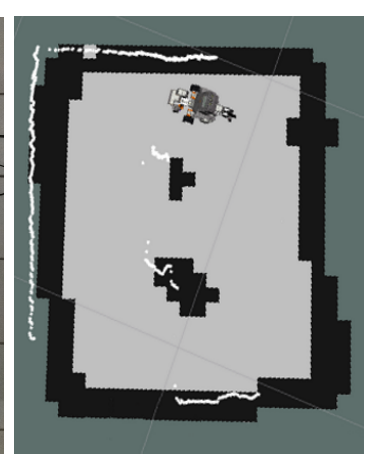

(c) Mapa gerado.

Figura 5. Resultados obtidos referentes a localização e mapeamento durante as práticas propostas.

materiais de apoio, incluindo tutoriais e códigos utilizados para a implementação da disciplina estão disponíveis nos links apresentados ao longo do artigo.

A metodologia de ensino proposta vem sendo aplicada desde o segundo semestre de 2018 na disciplina optativa Laboratório de Robótica Móvel, oferecida como "Tópicos Especiais" para alunos de graduação dos cursos de Engenharia de Controle e Automação, Elétrica e Sistemas da Universidade Federal de Minas Gerais (UFMG). Até o presente momento, 11 alunos já cursaram a disciplina.

Apesar de recente, esta já vem apresentando bons resultados. Após adquirir conhecimentos e experiência com o ROS, dois alunos que cursaram a disciplina (co-autores Israel e Lorran) viraram bolsistas de iniciação científica e tecnológica do projeto "Dispositivo Robótico para Inspeção de Ambientes Confinados" (Santos et al., 2018; Cota et al., 2018), realizado em parceria com o Instituto Tecnológico Vale (ITV). Além disso, os resultados das práticas propostas foram demonstrados no evento Mostra Sua UFMG 2019, como forma de apresentação e motivação do curso de Engenharia de Controle e Automação para alunos do ensino médio.

Como trabalho futuros, atividades complementares relacionadas ao seguidor de linha serão introduzidas na disciplina, incluindo novas formas de utilização e calibração dos sensores de luz, e técnicas de controle dos motores incluindo controladores PI e PID, Fuzzy e Aprendizagem por Reforço.

\section{REFERENCIAS}

Choset, H.M., Hutchinson, S., Lynch, K.M., Kantor, G., Burgard, W., Kavraki, L.E., and Thrun, S. (2005). Principles of robot motion: theory, algorithms, and implementation. MIT press.

Cota, E., Rocha, F., Perez, H.I., and Freitas, G. (2018). Avaliação de técnicas de odometria aplicadas a um dispositivo robótico móvel. In Congresso Brasileiro de Automática - CBA2018. João Pessoa.

Doucet, A., De Freitas, N., Murphy, K., and Russell, S. (2000). Rao-blackwellised particle filtering for dynamic bayesian networks. In Proceedings of the Sixteenth conference on Uncertainty in artificial intelligence, 176-183. Morgan Kaufmann Publishers Inc.

Dudek, G. and Jenkin, M. (2010). Computational principles of mobile robotics. Cambridge university press.

Ferguson, D., Morris, A., Haehnel, D., Baker, C., Omohundro, Z., Reverte, C., Thayer, S., Whittaker, C., Whittaker, W., Burgard, W., et al. (2004). An autonomous robotic system for mapping abandoned mines. In Advances in Neural Information Processing Systems, 587-594.
Grisetti, G., Stachniss, C., Burgard, W., et al. (2007). Improved techniques for grid mapping with rao-blackwellized particle filters. IEEE transactions on Robotics, 23(1), 34.

Hasan, K.M., Abdullah-Al-Nahid, and Mamun, A.A. (2012). Implementation of autonomous line follower robot. In 2012 International Conference on Informatics, Electronics Vision (ICIEV), 865-869. doi:10.1109/ICIEV.2012.6317486.

Ishigami, G., Nagatani, K., and Yoshida, K. (2007). Path planning for planetary exploration rovers and its evaluation based on wheel slip dynamics. In Robotics and Automation, 2007 IEEE International Conference on, 2361-2366. IEEE.

Júnior, C., Naves, J.A., D'Angelo, T., and Monteiro, P.M.d.B. (2014). Experiência de aprendizagem baseada em projetos no ensino de robótica. Versión Abierta Español-Portugués, 183.

Kaiser, F., Islam, S., Imran, W., Khan, K.H., and Islam, K.M.A. (2014). Line follower robot: Fabrication and accuracy measurement by data acquisition. In 2014 International Conference on Electrical Engineering and Information Communication Technology, 1-6. doi:10.1109/ICEEICT.2014.6919137.

Murphy, K.P. (2000). Bayesian map learning in dynamic environments. In Advances in Neural Information Processing Systems, $1015-1021$.

Nagatani, K., Kiribayashi, S., Okada, Y., Otake, K., Yoshida, K., Tadokoro, S., Nishimura, T., Yoshida, T., Koyanagi, E., Fukushima, M., et al. (2013). Emergency response to the nuclear accident at the fukushima daiichi nuclear power plants using mobile rescue robots. Journal of Field Robotics, 30(1), 44-63.

Pakdaman, M. and Sanaatiyan, M.M. (2009). Design and implementation of line follower robot. In 2009 Second International Conference on Computer and Electrical Engineering, volume 2, 585-590. doi:10.1109/ICCEE.2009.43.

Quigley, M., Conley, K., Gerkey, B., Faust, J., Foote, T., Leibs, J., Wheeler, R., and Ng, A.Y. (2009). Ros: an open-source robot operating system. In ICRA workshop on open source software, volume 3, 5. Kobe, Japan.

Santos, A.S., Azpúrua, H.I.P., Pessin, G., and Freitas, G.M. (2018). Path planning for mobile robots on rough terrain. In 2018 Latin American Robotic Symposium, 2018 Brazilian Symposium on Robotics (SBR) and 2018 Workshop on Robotics in Education (WRE), 265-270. IEEE.

Siciliano, B. and Khatib, O. (2016). Springer handbook of robotics. Springer.

Spong, M.W., Hutchinson, S., Vidyasagar, M., et al. (2006). Robot modeling and control.

Thrun, S. and Leonard, J.J. (2008). Simultaneous localization and mapping. Springer handbook of robotics, 871-889.

Wood, R.J. (2008). Robotic manipulation using an open-architecture industrial arm: a pedagogical overview [education]. IEEE Robotics Es Automation Magazine, 15(3), 17-18. 